www.jmscr.igmpublication.org

Impact Factor 5.84

Index Copernicus Value: 83.27

ISSN (e)-2347-176x ISSN (p) 2455-0450

crossref DOI: https://dx.doi.org/10.18535/jmscr/v5i2.40

Journal Of Medical Science And Clinical Research

IGM Publication

An Official Publication of IGM Publication

$\underline{\text { Original Article }}$

\title{
Comparative Study of Demonstration of Mycobacterium Tuberculosis on Tissue by Concentration Bleach Method and Auramine Rhodamine with Ziehl Neelsen Staining- An Autopsy Study
}

\author{
Authors \\ Anjali Bode ${ }^{1}$, Vikas Kavishwar ${ }^{2}$ \\ ${ }^{1}$ Assistant Professor, ${ }^{2}$ Associate professor \\ Department of Pathology, Indira Gandhi Govt Medical College, Nagpur \\ Corresponding Author \\ Dr Anjali N Bode \\ 401 Shivali app Renge layout Trimurti Nagar, Nagpur INDIA \\ Email:anjalidhote@ rediffmail.com, Mobile No+91-9421942423
}

\begin{abstract}
Objectives: Diagnosis of tuberculosis is depending upon to demonstrate the bacilli in tissue section or cultivate the bacilli. Objectives of our study were to compare demonstration of acid fast bacilli by modified concentration bleach method and Auramine Rhodamine fluorescent stain with conventional Ziehl-Neelsen staining on autopsy tissue material.

Method: Study was conducted in tertiary care hospital in Mumbai of India.It was three year prospective autopsy study.Total 887 clinical autopsies had been performed during three year. Out of them 108 cases were of tuberculosis. Tissue from tubercular lesions were subjected for routine Ziehl-Neelsen stain, concentration bleach and Fluorescent staining. Chi square test was applied for significance of study.

Results: Out of 108 cases proportion of acid fast bacilli positive cases were maximum by Auramine Rhodamine staining method $74 \%$, followed by concentration bleach method $45 \%$ and conventional Ziehl Neelsen staining $25 \%$.

Conclusions: Fluorescent staining has more advantages than concentration bleach and Ziehl Neelsen staining method in detecting tubercular bacilli. Still institutes who don't have fluorescent microscope can used concentration bleach method because it definitely increases rate of positivity on tissue.

Keywords- autopsy tissue, tuberculosis, Ziehl Neelsen stain, Concentration bleach method, Auramine Rhodamine fluorescent stain.
\end{abstract}

\section{Introduction}

Tuberculosis is one of the oldest diseases known to humanity and among the most deadly epidemics. One third of world's population is infected with mycobacterium tuberculosis and 9.6 million new cases occur each year. Nearly 1.45 million people die annually due to tuberculosis making it the leading cause of death due to infectious agent worldwide. ${ }^{(1)}$

Incidence of tuberculosis in developing countries like India has dramatically increased over few decades and having highest burden of TB with estimated incidence of 2.2 million cases out of global incidence. ${ }^{(2)}$ Also contributing to it is the 
human immunodeficiency virus. Nearly every year 2.1 million people infected with HIV in India and approximately 0.35 million people infected with tuberculosis which is cause of death. ${ }^{(1)}$

Considering the load of tuberculosis infection, early diagnosis is essential to limit the morbidity and mortality. In autopsy cases one need to give a definitive cause of death in patient who died of tubercular features. Only full proof diagnostic criteria are demonstration of tubercular bacilli on tissue.

Identification of mycobacterium is essential for a definitive diagnosis; however, culture takes weeks for identification, and in formalin fixed tissue, bacilli died so culture is not possible. ${ }^{(3,4)}$ There are newer molecular technique such as PCR for identification of bacilli on formalin fixed paraffin embedded tissue . ${ }^{(5,6,7)}$ But molecular technique is costly for routine use in developing countries such as India where tuberculosis is prevalent. ${ }^{(8)}$

So diagnosis is made by using conventional Ziehl Neelsen (Z-N) staining on tissue. But it is found that in formalin fixed tissue the efficacy of conventional $\mathrm{Z}-\mathrm{N}$ stain method is reduced as compare to aspiration material. Hence give false negativity. To overcome this there are many other methods which help in detecting more positive cases, such as concentration bleach method, Auramine - Rhodamine fluorescent staining .

Modified Concentration bleach method and Auramine - Rhodamine (AR) fluorescent staining these both methods are safe and inexpensive. But in AR Staining one need florescent microscope and expertise for detecting bacilli.

Basically, this study was an attempt to find out cost effective, rapid, and sensitive technique which can be used routinely in developing countries for diagnosis on tissue. The study

demonstrated the correlation of the histomorphological features with various techniques on formalin fixed tissue from autopsies. We tried to use AR staining and modified concentration bleach method to detect Mycobacterium tuberculosis and compare it with conventional $\mathrm{ZN}$ stain on tissues.

\section{Material and Method}

It is three year prospective clinical autopsies study in tertiary center of Mumbai in India. During three year total 887 complete clinical autopsies had been performed out of which 108 cases were clinically diagnosed as tuberculosis.

In 108 cases all available organs were examined for tubercular lesions. Lesions were recorded with descriptive notes. Lesions with fibrocavitory with caseation necrosis were selected and necrotic tissue from fresh as well as formalin fixed was taken and divided in two samples. One tissue sample proceeded for concentration bleach method other sample for routine histopathology, $\mathrm{ZN}$ and AR fluorescent stain.

For concentration bleach method we took necrotic tissue, we crushed that tissue and then proceeded. In crushed necrotic tissue we added $1 \mathrm{ml}$ commercially available $4 \%$ sodium hypochlorite. We mixed it thouroughly. Initially we incubate for 30 min but did not get satisfactory results. So we increased time period for incubation from 30 minutes to $1 \mathrm{hr}$ to $1.30 \mathrm{hr}$ to $2 \mathrm{hr}$ to overnight. Equal volume of commercially available distilled water was then added and mixture was centrifuged at 3000rpm for 15 minutes. Supernatant was discarded, and smear was prepared using one drop sediment, air dried, heat fixed and stained by Ziehl - Neelsen stain. Material from different incubation were stained and studied.

Other necrotic tissue was proceeded for routine histopathology; slides were made and stained for $\mathrm{H} \& \mathrm{E}, \mathrm{Z}-\mathrm{N}$ and AR stain

Auramine Rhodamine staining method - tissue was deparaffinised, filtered commercially available Auramine Rhodamine solution was poured on section and kept for 10 min then section was washed in tap water and differentiated in $0.5 \%$ hydrochloric acid in alcohol, again washed in tap water for $2 \mathrm{~min}$. Counterstained with $0.5 \%$ potassium permanganate for $2 \mathrm{~min}$, washed in tap water and blot dry. Dehydrated in absolute alcohol for 15 seconds.

Cleared in xylol. Cover slipped using D.P.X. mounting media.. Slides examined under high 
power $(\times 400)$ which was confirmed under oil immersion $(\times 1000)$.

AFB appears as yellow to orange, slender, rod shaped bacilli under fluorescent microscopy. Smears stained by concentration bleach method and $\mathrm{ZN}$ method were directly examiner under oil immersion (X1000) using light microscopy and appear long slender, beaded, pink bacilli.

We used the CDC laboratory guideline for reporting acid fast bacilli for $\mathrm{Z}-\mathrm{N}$ and $\mathrm{A}-\mathrm{R}$ staining. (9)

\section{Result}

Over the three year study we found there was successively decreasing in no of autopsies. Out of 108 cases 36 cases were HIV positive in which all serological reports were avaliable. Male preponderance were noted accounting for $64 \%$ (69 / 108) of cases.

Out of 108 cases 61 cases shows both pulmonary and extra pulmonary lesions. Predominately pulmonary lesion was fibrocavitory with caseation (fig1) $48 \%$ ( 51/108) followed by miliary $41 \%$. In extra pulmonary lymph nodes showed preponderance $96 \%$ (58/61 ) followed by liver, spleen and brain, intestine, kidney and adrenal. ${ }^{(10,11)}$

Paraffin embedded $\mathrm{H}$ \& $\mathrm{E}$ stained slides were studied. Microscopically they were divided in three patterns. ${ }^{(12)}$

Reactive pattern shows well formed granuloma without caseation in 7\% of cases .In this pattern acid fast bacilli were absent or rare.(Fig 1b) Hypo reactive pattern shows less well formed granuloma with area of necrosis having highest percentage $64 \%$ of cases .(Fig 1c) in this pattern acid fast bacilli were 10 to $100 / 100$ high power field.

Non reactive pattern in which there were irregular areas of caseous necrosis along with nuclear debris was seen in $29 \%$ cases. (Fig 1d) Number of bacilli was more than $100 / 100$ high power field.

Out of 108 cases $25 \%$ (27) were positive by Z-N staining and showed positivity from $1+$ to $4+$. 4+ positivity seen in non reactive pattern (Fig2a). By concentration bleach method there was definitely increased in no for detection of bacilli from $45 \%$ (49) (Fig2b). We got satisfactory result with overnight incubation. It was found with overnight fixation there was reduction in debris which help in improving detection of tubercle bacilli. Out of 108 cases 80 were positive by AR fluorescent staining i.e. $74 \%$ ( Fig 2c) which is highly significant.

8 cases which showed reactive pattern were negative by both concentration bleach and Z-N staining method. But out of this 3 cases were positive with A-R stain. These 3 cases were HIV positive.

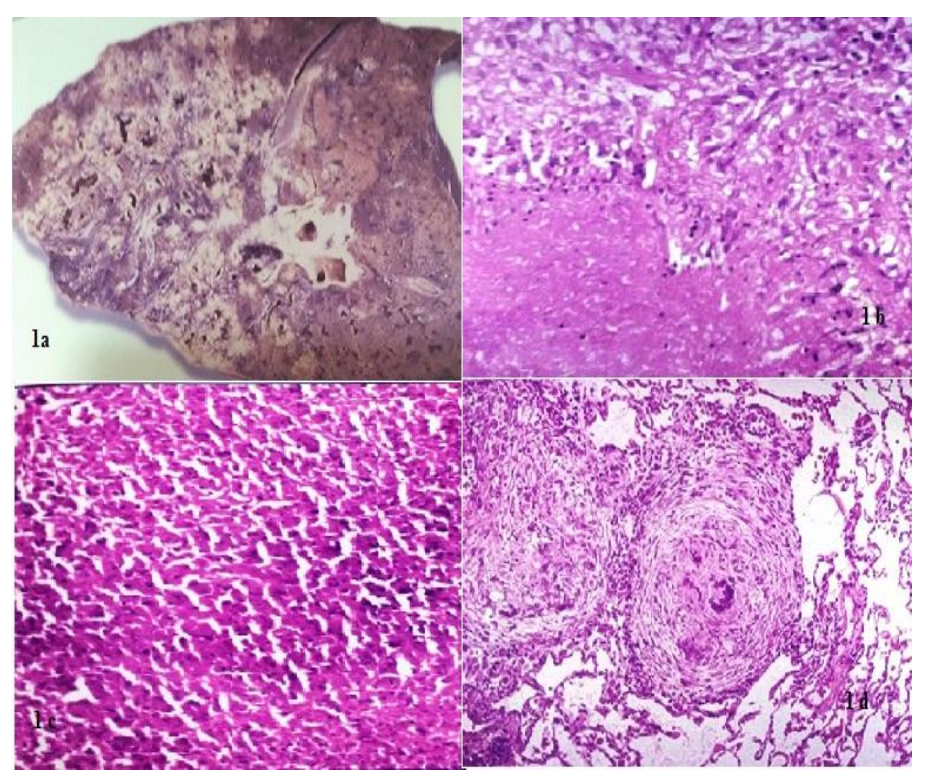

$\mathbf{1 a}$ - gross of lung showing fibrocavitory lesion

1b - H\&E stained section shows caseating granuloma - hyporeactive pattern (40x)

1c- H\&E stained section shows only caseation necrosis and degenerated polymorphs - Non reactive pattern $(40 \mathrm{x})$

1d- H\&E stained section shows well formed granuloma without caseation - Reactive pattern (40x) 


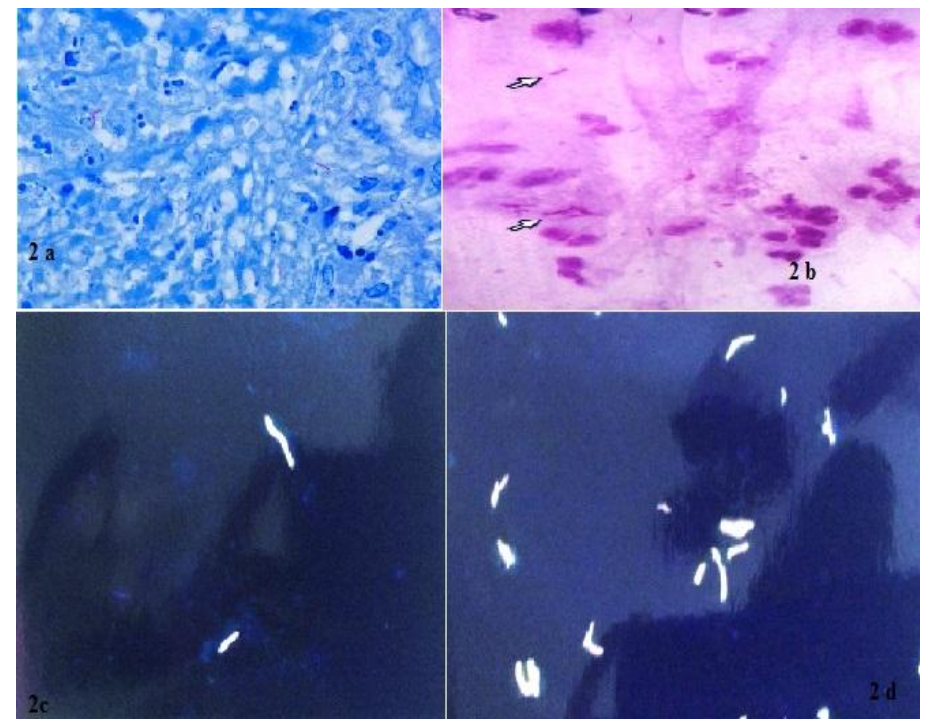

2a $-\mathrm{ZN}$ stain tissue section shows pink coloured beaded bacilli (100x)

2b- shows pink bacilli stained by concentration bleach method (100x)

2c \& 2d - AR fluorescent stain show golden yellow slender bacilli (100x)

\section{Discussion}

Tuberculosis has always been a major problem in India and has many ongoing research and demonstration of bacilli projects on TB. ${ }^{(13)}$

The detection of AFB is often considered as the evidence of the infected state. Thus, the laboratory plays a critical role in the diagnosis of TB. ${ }^{(14)}$ In developing countries like India financial constraint, rapid and inexpensive diagnostic methods assume great importance in tuberculosis control.

Due to peculiar disease process, mycobacterium are often difficult to demonstrate in cases of tuberculosis so in the early 1940s, the comparison of the fluorescent method and concentration bleach method with the conventional ZN method on sputum smears was implemented to improve the smear positivity for the detection of AFB. The use of its increased sensitivity and ease of interpretation compared with the $\mathrm{ZN}$ method. ${ }^{(15)}$ Mycobacterium culture is time consuming and of no use in non viable bacilli in formalin fixed tissue.

Use of concentration bleach method is known in sputum and FNAC material. Its use on tissue is underreported. The present study was our attempt to use the concentration bleach and fluorescent method and compare it with the conventional $\mathrm{ZN}$ method on autopsy tissue materials. For concentration bleach method we increased incubated time for overnight. In cases of sputum and FNAC material incubation for $30 \mathrm{~min}$ to $1 \mathrm{hr}$ is given in literature. ${ }^{(16,17)}$ But we found difficulty with less incubation period so we increased incubation time that may cause degradation of necrotic tissue and concentrate tubercular bacilli. By doing, so not significantly but definitely there was increased in no of positive cases as compared to $\mathrm{ZN}$ stain from $25 \%$ to $43 \%$ cases. On searching literature all studies are on sputum and on FNAC material and in all studies it was found concentration bleach method definitely increases detection rate for tubercle bacilli. ${ }^{(16,17,18)}$

The staining with AR increased the positivity by $47 \%$ over the conventional $\mathrm{ZN}$ method and by $29 \%$ over concentration bleach method. The AFB typically fluoresces as golden, slender, curved, rod-shaped bacilli. However, good observation is required to distinguish with certainty AFB from other small, naturally fluorescent particles present in some smears. When first using fluorescent microscopy, it is necessary to examine all small fluorescent objects seen both with the $\times 10$ and $\times 40$ objectives. With practice, it becomes possible to distinguish bacilli with a fair degree of certainty under the $\times 10$ objective only, so that almost all negative smears can be examined with this objective only. However, it is always necessary to confirm the bacillary morphology with the higher power when the sections contain a low density of bacilli.

Same as concentration bleach method there are relatively few reports of Auramine Rhodamine staining on formalin fixed tissue present. Many studies are present on sputum and cytology materials. ${ }^{(19.20,21)}$ In our study there were $74 \%$ cases detected by AR stain. 8 cases showed reactive pattern and all this 8 cases were negative by $\mathrm{ZN}$ and concentration bleach method due to paucity of bacilli. Out of 8 cases 3 were positive by AR stain because though there was low 
densities of bacilli that can be fluoresce by AR stain and easily picked up. But in such cases we need lot of practice and observation to give positivity otherwise can be reported as false positivity.

We concluded that concentration bleach method is complementary to $\mathrm{ZN}$ staining and can improve detection of acid fast bacilli on tissue. It is easily available and inexpensive. AR fluorescent method was significantly superior and sensitive than concentration bleach and $\mathrm{ZN}$ method. This is particularly useful in formalin fixed tissue where culture is not possible also fluorescent stained slide can be examined under low magnification allowing for much larger areas for section to be examined in a short period of time. It has great diagnostic value in patients with low density of bacilli that are likely to be missed on $\mathrm{ZN}$ stain.

No separate financial support

\section{Contribution Details}

Dr. Anjali Bode - Data acquisition, literature search, Manuscript preparation

Dr. Vikas Kavishwar - Concept, Design, Manuscript preparation

\section{References}

1. World Health Organisation .Global tuberculosis report 2016, annexure 4. WHO Geneva 2016.

2. TB India 2016 Revised National TB control Programme Annual Status Report, New Delhi , 2016.

3. Deniz Demiryurek, Alp Bayramoglu, Semsettin Ustacelebi : Infected agents in fixed human cadavers : A brief review and suggested guidelines ; The Anatomical Record 2002,269.194-197.

4. Kappel TJ, Reinartz JJ, Schmid JL, Holter JJ, Azar MM. The viability of Mycobacterium tuberculosis in formalinfixed pulmonary autopsy tissue : review of the literature and brief report. Hum Pathol. 1996 Dec;27(12):1361-4.
5. Isik Somuncu Johansen, Vibeke Ostergaard Thomsen, Arne Forsgren,Birgit Fischer Hansen, Bettina Lundgren: Detection of Mycobacterium tuberculosis Complex in Formalin - Fixed, ParaffinEmbaded Tissue Specimens with Necrotising Granulomatous Inflammation by Strand Displacement Amplification ; J Mol Diagn 2004,6,231-236.

6. Salian NV, Rish JA, Eisenach KD, Cave MD, Bates JH. Polymerase chain reaction to detect Mycobacterium tuberculosis in histologic specimens. Am J Respir Crit CareMed. 1998;158:1150-1155. [PubMed]

7. Diaz ML, Herrera T, Lopez-Vidal Y, Calva JJ, Hernandez R, Palacios GR, Sada E. Polymerase chain reaction for the detection of Mycobacterium tuberculosis DNA in tissue and assessment of its utility in the diagnosis of hepatic granulomas. $\mathbf{J}$ Lab Clin Med 1996;127:359 363. [Pub Med]

8. Savic B, Sjobring U, Alugupalli S, Larsson L, Miorner H. Evaluation of polymerase chain reaction, tuberculostearic acid analysis, and direct microscopy for the detection of Mycobacterium tuberculosis in sputum. J Infect Dis. 1992; 166:117780. [PubMed]

9. Kent PT, Kubica GP: Public Health Mycobacteriology: A guide for the Levell III laboratory. Atlanta Department of Health And Human Services, 1985, 48-51.

10. Lee MP, Chan JW, Ng KK, Li PC. Clinical manifestations of tuberculosis in HIV-infected patients. Respirology 2000; 5: 423-6.

11. S K Sharma, A Mohan : Extra pulmonary tuberculosis: Indian J Med Res 2004,120, 316-353

12. Smith MB, Boyars MC, Veasey $\mathrm{S}$ : Generalised Tuberculosis In the Acquired Immune Deficiency Syndrome - A Clinicopathological Analysis Based On 
Autopsy Finding : Arch pathol Lab Med 2000, 124, 1267-1274.

13. Central tuberculosis division. Tuberculosis - A guide for practising physicians. New Delhi: Revised National Tuberculosis Control Programme, Directorate General of Health Services; 2004. 1-5.

14. American thoracic society. Diagnostic standards and classification of tuberculosis. Am Rev Respir Dis. 1990; 142:725-35. [PubMed]

15. Holst E, Mitchison DA, Radhakrishna S. Examination of smears for tubercle bacilli by fluorescent microscopy. Indian J Med Res. 1959;47:495-9.[Pub med]

16. Gangane N, Anshu, Singh R. Role of modified bleach method in staining of acid-fast bacilli in lymph node aspiration. Acta Cytol. 2008;52:325-8. [PubMed]

17. B Chandrashekhaar, Aruna Prayaga: Utility of concentration method by modified bleach technique for the demonstration of acid-fast bacilli in the diagnosis of tuberculous lymphadenopathy; J Cytol,2012,29 ;165-168.

18. Preeti Singh, Monika Rathi, Nidhi Verma, Neha Singh, Virendra Tapparwal ; Modified bleach method for detection of acid-fast bacilli in lymph node aspirates and its comparison with the conventional Ziehl - Neelsen stain : Int J Adv Med and Health Res, 2015;2:83-86.

19. Vamseedhar Annam, Mohan H Kulkarni, and Rekha B Puranik Comparison of the modified fluorescent method and conventional Ziehl-Neelsen method in the detection of acidfast bacilli in lymphnode aspirates; Cytojournal 2009, 6:13.

20. H. Silver, A. C. Sonnenwirth, and N. Alex; Modifications in the fluorescence microscopy technique as applied to identification of acid-fast bacilli in tissue and bacteriological material: J Clin path, 1966; 19:583.
21. $\mathrm{Ba} \mathrm{F}^{1}$, Rieder HL A comparison of fluorescence microscopy with the ZiehlNeelsen technique in the examination of sputum for acid-fast bacilli ; Int $\mathbf{J}$ TubercLung Dis1999, 3:1101-05. 